\title{
Right Ventricular Cardiogenic Shock Caused by Myocardial Infarction: A Review
}

\author{
Luhanda Leonora Cardoso Monti Sousa ${ }^{1 *}$, Guilherme Dagostin de Carvalho', Amália Bonfogo ${ }^{2}$, Sasha \\ Barbosa da Costa Pimenta Duarte ${ }^{1}$ and Thiago Luis Scudeler ${ }^{1}$ \\ ${ }^{1}$ Instituto do Coração (InCor), Hospital das Clínicas da Faculdade de Medicina da Universidade de São Paulo, São Paulo-Brazil \\ ${ }^{2}$ Instituto do Coração de Santa Catarina, Brazil
}

*Corresponding author: Luhanda Leonora Cardoso Monti Sousa, Instituto do Coração (InCor), Hospital das Clínicas da Faculdade de Medicina da Universidade de São Paulo, São Paulo-Brazil

Received Date: May 04, 2020

Published Date: May 20, 2020

\begin{abstract}
Left ventricular inferior wall acute myocardial infarction is associated with right ventricular myocardial infarction (RVMI) in up to $50 \%$ of cases. The worse prognosis of RVMI is due to the greater propensity for refractory cardiogenic shock (CS) when compared to isolated inferior wall myocardial infarction (IWMI). The diagnosis is based on the clinic, biomarkers, electrocardiogram (ECG) and coronary angiography. Meanwhile, imaging modalities, such as transthoracic echocardiography (TTE) and cardiac magnetic resonance (CMR) play a complementary role. Understanding ventricular interdependence is of paramount importance to perception and management hemodynamic changes. In this scenario, hemodynamic impairment can be caused by right ventricular (RV) dysfunction, left ventricular (LV) dysfunction, a combination of these, or even by other disorders. Early revascularization is the cornerstone of treatment. When cardiac output (CO) and blood pressure do not improve with the initial volume expansion, the introduction of vasopressors and inotropes is necessary. For those who survive the acute phase, long-term prognosis is usually favorable. The best prognosis in these cases relies on early revascularization and adequate hemodynamic management.
\end{abstract}

\section{Introduction}

Left ventricular inferior wall acute myocardial infarction is associated with RVMI in up to $50 \%$ of cases, and occasionally, with infarction of the anterior territory [1-4]. It has a worse short-term prognosis due to a greater propensity for CS, when compared to isolated IWMI. However, in patients who survive the acute phase, long-term prognosis is usually favorable, depending on early revascularization and adequate hemodynamic management [1-3]. The involvement of the RV is commonly underdiagnosed despite its high prevalence [1,2]. High-grade trioventricular block difficults the hemodynamic management, especially when associated with inadequate preload and previous LV dysfunction. The diagnosis is based on clinical features, biomarkers, ECG and coronary angiography, while imaging modalities, such as TTE and CMR, play a complementary role [1-4]. Early revascularization, whether by percutaneous coronary intervention (PCI) or chemical fibrinolysis, remains the cornerstone of treatment. The maintenance of an adequate preload, as well the use of inotropes and vasopressors, when appropriated, are extremely important [1-3]. Some cases will require mechanical circulatory assistance, including cardiac assistance devices, percutaneous cardiopulmonary support and an intra-aortic balloon pump (IABP) [5,6]. The use of a pulmonary artery catheter (CAP) is indicated in refractory cases or diagnostic doubt [5]. This paper aims to review the relationship between early diagnosis and revascularization in the prognosis in the short and long term, as well as the implications of underdiagnosis in the associated RVMI.

\section{Anatomy and Physiology}

The normal RV is anatomically and physiologically different from the LV. Its arterial blood supply derives from the right coronary artery (RCA). It has a half-moon shape, and a thinner fee wall (3-5 mm in adults), although it is larger in volume (10 to $15 \%$ ) compared to the LV. Both ventricles share the same interventricular septum (IVS) and are surrounded by the same pericardium [7]. RV 
is arranged between two low pressure venous systems [6]. The adaptation of the RV to the disease has been classified according with volume and pressure overload as well as commitment of its contractility that occurs in ischemia. So far, there aren't consistent data in humans that can establish differences in the mechanisms of myocardial contractility between RV and LV, and the genetic differences are less studied. Proteomic and transcriptomic studies suggest great overlap with the contraction and excitation process, and it is safe to assume similarity between these functions [6].

Some peculiarities of the RV make it more resistant to ischemia than the LV. The chronic right heart failure attributable to RVMI is rare. Because of its low afterload, considering that its pulmonary vascular resistance is one tenth of the systemic vascular resistance, $\mathrm{RV}$ has what we call ischemic myocardial conditioning. In this situation the RV has oxygen consumption and extraction rates at rest significantly lower than the LV [6-8]. In periods of high metabolic demand, such as ischemia, the RV can increase its extraction rate, rather than of the coronary flow [6]. In addition, in the absence of severe RV hypertrophy, it receives irrigation in systole and diastole and a large network of collaterals through those of the left coronary arteries, so that even in the situation of chronic RCA occlusion, the $\mathrm{RV}$ is preserved [6-8].

\section{Coronary irrigation}

RVMI accompanies the IWMI about $30-50 \%$, since both territories are irrigated by the RCA, which is dominant in $85 \%$ of cases. However, in the $15 \%$ of the general population where dominance the left circulation or in those with chronically occluded proximal RCA, more than $50 \%$ of the RV free wall is supplied by the left collateral circulation, so that the culprit artery could be an left anterior descending artery (LAD) or circumflex artery (CxA). The CxA will be to blame in most of these cases [1-3,7].

\section{Ventricular interdependence}

The concept of ventricular interdependence is of huge importance for understanding the hemodynamic changes caused by RVMI. Ventricular interdependence assumes that the LV cardiac output directly depends on the RV's ability to manage its preload and send it to the left cavities. The IVS and the pericardium are an essential part of this process. In the absence of severe RV and LV dysfunction, this process occurs in a harmonious manner $[7,9]$. The hemodynamic consequences of RVMI depend on the extent of the dysfunction of the RV free wall and the simultaneous involvement of the LV [1,2,9]. An important RV perfusion deficit usually occurs in proximal RCA occlusions and is responsible for severe hemodynamic manifestations, which happen in less than $50 \%$ of RVMI's patients [7]. In these cases, ischemia is capable of leading to a decrease in the overall performance of the RV, due to the dyskinesia of its free wall and the ischemia of the right atrium concurrently $[7,10]$. The ischemic RV is rigid, dilated and, when associated with ischemia of the right atrium, has its diastolic filling limited. Consequently, the blood supply to the LV becomes progressively scarce. During diastole, the RV moves the IVS towards the LV, which is already deprived of volume, further impairing filling and reducing compliance. The LV depends on the RV to obtain its preload and the RV depends on the integrity of the contraction between the LV and the IVS to perform its function [1-3,10].

In isolated RVMI, the performance of the RV is preserved, since the IVS-LV contraction remains intact, presaging a better prognosis. In the RVMI associated with the IWMI, the IVS is injured and this compromises biventricular contraction, leading to the lack of compensation that had to have been transmitted by the ventricular interaction during the systole, through the septal thickening, that now is absent. Consequently, RV emptying is compromised, causing an increase in filling pressures and diastolic dysfunction (Figure 1). So, the evolution to hypotension and low cardiac output, refractory to the initial volume load is inevitable $[1,8,10]$. Subsequent impairment of coronary perfusion leads to the spread of biventricular systolic and diastolic dysfunction and rapid progression to cardiogenic shock, although LV systolic function remains intact $[8,10]$. Abrupt dilation of the $\mathrm{RV}$ in the non-uniform pericardium increases intra-pericardial pressure and further restricts the filling and complacency of both ventricles, in addition to intensifying the diastolic interactions mediated by IVS $[7,9]$.
A

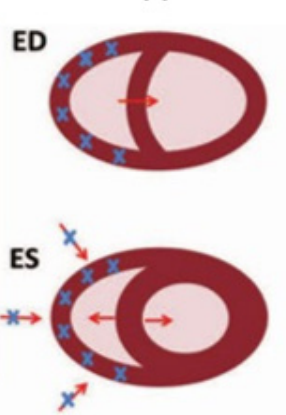

B

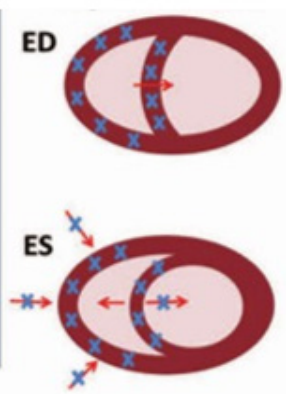

Figure 1: A) Isolated RVMI; B) RVMI with septal ischemia. Adapted from Inohara, et al. [1]. ED = end-diastole; $E S$ = end-systole.

\section{Hemodynamic repercussions}

It should be borne in mind that in patients with RVMI associated with IWMI hemodynamic impairment can be caused by RV dysfunction, LV dysfunction, a combination of these or other disorders (for example, rupture of the IVS or tricuspid regurgitation). In some cases, the distinction of the relative participation of the $\mathrm{LV}$ or RV may be uncertain and invasive hemodynamic monitoring with a pulmonary artery catheter (PAC) may be necessary both to understand the pathophysiology and to guide treatment [8]. Hypoperfusion becomes clinically relevant when the cardiac index (CI) falls below 2.2 liters $/ \mathrm{min} / \mathrm{m}^{2}$. If the pulmonary capillary pressure (PCP) is above $18 \mathrm{mmHg}$, the clinical status of the patient reflects these subgroups, however, until $25 \%$ of 
patients with CI below 2.2 liters $/ \mathrm{min} / \mathrm{m}^{2}$ and $15 \%$ of patients with PCP above $18 \mathrm{mmHg}$, aren't clinically recognized [7,8].

The hemodynamic consequences of RVMI include a combination of findings that indicate RV systolic and diastolic dysfunction. These changes are usually low $\mathrm{CI}$ and a disproportionate increase in the RV filling pressure, when compared to the LV filling pressures, which in general are reduced [7]. The isolated RVMI, high filling pressures in the right chambers, such as central venous pressure (PVC) and right atrium pressure (RAP), with normal or low PCP would be expected and, when LV function is preserved, the CI tends is normal. However, in the presence of concomitant IWMI, an increase in PCP is observed, generally above $18 \mathrm{mmHg}$ and low $\mathrm{CI}$ [11]. The objectives of hemodynamic therapy include maintaining cardiac performance, blood pressure and protection of the myocardium at risk.

\section{Clinical manifestations}

Initial manifestations may be similar as seen in other scenarios of myocardial infarction. In the case of RVMI with IWMI, the typical triad observed on physical examination is hypotension, especially after the administration of small doses of nitrate, jugular stasis and clean lung auscultation. However, this triad has high specificity (96\%), but very low sensitivity (25\%) [9]. Kussmaul's venous signal (distention of the jugular vein on inspiration), a characteristic of constrictive pericarditis, also can occur in RVMI. The murmur of tricuspid regurgitation may be present. The presence of a paradoxical pulse (drop in systolic pressure above 10 mmHg on deep inspiration) and a Kussmaul's sign in the context of lower IWMI are highly predictive signs of associated RVMI $[12,13]$. In some cases, these symptoms are not present on admission and do not occur until diuretics or nitrates are administered [13].

\section{Diagnosis}

The initial diagnosis of RVMI is made with physical examination, cardiac biomarkers, ECG and corroborated by cardiac catheterization that shows the culprit artery [13-16]. Non-invasive imaging modalities play a complementary role. However, when there is diagnostic doubt or in cases of refractory cardiogenic shock, invasive hemodynamic monitoring with CAP becomes necessary and ventricular filling pressures can also be measured in the cardiac catheterization.

\section{Electrocardiogram}

The 12-lead ECG demonstrates ST segment elevation in the inferior wall $>1 \mathrm{~mm}$ in two or more contiguous leads and the $>0,5$ $\mathrm{mm}$ elevation in right precordial leads. Determining whether the $\mathrm{T}$ wave is positive or negative in right leads helps to distinguish whether the occlusion is proximal or distal to the RCA versus the occlusion of the CxA [7].

\section{Echocardiogram}

Echo is usually the initial imaging exam and can be performed at the bedside in unstable patients. The most relevant findings include dilation and systolic dysfunction with changes in contractility (akinesias, hypokionesias or dyskinesias) of the RV free wall, as well as paradoxical movement of the IVS. In cases of concomitant IWMI, the LV function is preserved at the onset of the condition or slightly reduced. Pulmonary and/or tricuspid regurgitation corroborate the involvement of the RV. However, this examination has some technical limitations due to the complex shape of the RV, which may not be fully visualized in any 2D echo projection. Although not validated in acute situations, the volumes of three-dimensional echo are comparable to those from CMR $[16,17]$.

\section{Cardiac resonance}

CMR allows accurate characterization of ischemic RV. Studies of CMR demonstrate a large number of cases of RVMI in patients with IWMI (47-57\%) and some patients with previous myocardial infarction (11-65\%) with involvement of the RV to some extent. Two observational reports comparing RV involvement between different imaging modalities in acute IWMI indicated that RV involvement was significantly more detected by CMR than by ECG and TTE. However, the use of CMR in acute patients has not yet been established, remaining as an extremely accurate alternative for diagnosis and prognosis in stable patients $[12,17]$.

\section{Cardiac catheterization}

Cardiac catheterization has enabled the diagnosis and treatment of the culprit artery, as well as the assessment of pressure curves and venous pulse waveform. The culprit artery is often the RCA near the marginal branch. In patients with left dominance, CxA occlusion can also be found. Although uncommon, $\mathrm{RV}$ involvement may be present in patients with occlusion in the left anterior descending artery. Cabin, et al. studied 97 hearts with previous myocardial infarction and found that $13 \%$ were RVMI [18]. Is possible to perform the right cardiac evaluation and determine the hemodynamic diagnosis from the measurement of intracavitary pressures during catheterization. The diagnostic criteria were defined by Lopez-Sendon, et al. derived from a study of cardiac catheterization post-mortem autopsy, and included $\mathrm{RAP}>10 \mathrm{mmHg}$, RAP/PCP ratio $>0.8$ or RAP within $5 \mathrm{mmHg}$ of the PCP [19]. However, in cases of concomitant and significant LV dysfunction, the close relationship between RAP and PCP is not preserved, despite the fact that RAP remains elevated.

In the inspiration, the negative intrathoracic pressure increases venous return. This raises the blood volume for the RV and reduces it for the LV. However, RV dysfunction results in hypotension, low output and decreased ventricular compliance. What increases the filling pressures in the RV, leading to the loss of physiological inspiratory collapse, seen as Kussmaul's sign. The venous pulse waves are represented by a, c, x, v and y and reflect pressure abnormalities of the pathological RV. The RVMI may present with hemodynamic manifestations similar to those of constrictive pericarditis. The "a" and "v" waves, are equal in amplitude. The presence of a giant $\mathrm{v}$ wave, reflects the high filling RAP and the pronounced decrease $y$, configures the famous square root sign [7]. 


\section{Treatment}

Early revascularization remains a cornerstone in the treatment of RVMI and is associated with improved reduction of morbidity and mortality [3]. Bowers, et al. studied the outcomes and RV function using 2D TTE in 53 patients with RVMI before and after reperfusion therapy [20]. The result showed a dramatic recovery of $\mathrm{RV}$ function and excellent clinical results after early and complete RCA reperfusion using primary PCI. In contrast, unsuccessful reperfusion was associated with worse RV function, refractory CS and higher mortality rates. Early reperfusion was also crucial in preventing ventricular arrhythmias, which were seen much more frequently in patients with unsuccessful ones. RV ischemia can lead to systolic and diastolic dysfunction, deficit in LV preload and subsequent fall in the stroke volume. The establishment of an adequate preload in a compromised RV is crucial for maintaining an adequate cardiac output [21]. Initial therapy requires the administration of fluids, capable of promoting adequate filling of the $\mathrm{RV}$, raising the $\mathrm{CI}$, but without increasing its filling pressures. Vasodilator medications and intravascular volume depletors, such as nitrates and diuretics should be avoided. Generally, an initial challenge with $300 \mathrm{~mL}$ to $600 \mathrm{~mL}$ of $0.9 \%$ saline is recommended for 10 to 15 minutes [21,22]. However, some studies indicate that in certain cases, the increase in preload does not result in an increased CI [23-25]. This can be explained by the variability of initial volume status among patients and already established ventricular dysfunctions. In cases of euvolemic or hypervolemic patients, the RV preload can have exceeded its limit to maintain the $\mathrm{RV}$ to LV flow rate [7]. In these situations, monitoring with PAC is recommended, considering that excessive infusion of crystalloids is deleterious by promoting an increase in RV filling pressures. This reduces LV filling, compromises interventricular interaction, and leads to equalization of ventricular pressures due to increased intrapericardial pressure. Based on hemodynamic monitoring studies, it's not recommended to exceed a RAP or PCP of $20 \mathrm{mmHg}$ [22].

When $\mathrm{CO}$ and blood pressure do not improve with the initial volume expansion despite significant increases in RAP and PCP, the introduction of inotropes, such as dobutamine, is necessary. Dell'Italia, et al. demonstrated that the effect of dobutamine in patients with RVMI and refractory CS produced a statistically significant increase in systolic volume index [24]. Electrical stabilization, including heart rate control and maintenance of atrioventricular synchrony, is another key factor in preserving cardiac output in these cases. In addition, several extracorporeal support devices have been used to support RV failure secondary to infarction and have contributed to the improvement of RV shock $[26,27]$. In refractory CS patients, the IABP may be beneficial. Although does not directly influence RV performance, it can increase coronary perfusion and thus improve RV function, especially if the RCA has been recanalized. In addition, the performance of a RV with impaired function depends largely on the contraction of IVS and LV, which can be improved with the use of IABP [26]. In the last decades, several extracorporeal support devices have been developed with the premise of circumventing the RV failure and or the pulmonary circulation, in order to allow its recovery, and also acting in the LV failure, in cases refractory to initial clinical/ hemodynamic management [26], such as the percutaneous ventricular assist device Tandem Heart (Cardiac Assist Inc, USA), a centrifugal extracorporeal pump, which generates continuous flow with a minimal pulsating component of low amplitude. It provides a flow of up to $5.0 \mathrm{~L} / \mathrm{min}$ and pumps blood from the right atrium to the main pulmonary artery, bypassing an impaired RV. Kapur, et al. studied nine patients with refractory RV dysfunction and found that, although four patients died early, the percutaneous ventricular assist device was associated with significant hemodynamic improvement [26]. Veno-arterial extracorporeal membrane oxygenation (ECMO) systems can provide cardiac and respiratory support. These can be fully established through cannulation of a femoral artery and vein using the Seldinger technique and therefore, without surgery. In contrast to the RV auxiliary devices, this technique allows the total diversion of blood from the pulmonary bed and, therefore, does not cause additional elevation of pulmonary pressures, alleviating the RV overload, being useful in patients with obstructive hemodynamic pattern [26-28].

\section{Conclusion}

RVMI is often associated with IWMI. Adequate hemodynamic management represents a challenge when the diagnosis of RV involvement is uncertain. Patients with RV involvement require careful and continuous with Swan-Ganz catheter monitoring. Early diagnosis and revascularization with PCI or fibrinolysis remains the gold standard treatment, capable of to prevent progression to cardiogenic shock and effectively increase short- and long-term survival in these cases.

\section{Acknowledgement}

None.

\section{Conflict of Interest}

No conflict of interest.

\section{References}

1. Inohara T, Kohsaka S, Fukuda K, Menon V (2013) The challenges in the management of right ventricular infarction. Eur Heart J Acute Cardiovasc Care 2(3): 226-234.

2. Albulushi A, Giannopoulos A, Kafkas N, Dragasis S, Pavlides G, et al. (2018) Acute right ventricular myocardial infarction. Expert Rev Cardiovasc Ther 16(7): 455-464.

3. Kakouros N, Cokkinos DV (2010) Right ventricular myocardial infarction: pathophysiology, diagnosis, and management. Postgrad Med J 86(1022): 719-728.

4. Ondrus T, Kanovsky J, Novotny T, Andrsova I, Spinar J, et al. (2013) Right ventricular myocardial infarction: From pathophysiology to prognosis. Exp Clin Cardiol 18(1): 27-30.

5. van Diepen S, Katz JN, Albert NM, Henry TD, Jacobs AK, et al. (2017) Contemporary Management of Cardiogenic Shock: A Scientific Statement From the American Heart Association. Circulation 136(16): e232-e268. 
6. Sanz J, Sánchez-Quintana D, Bossone E, Bogaard HJ, Naeije R (2019) Anatomy, Function, and Dysfunction of the Right Ventricle. J Am Coll Cardiol 73(12): 1463-1482.

7. O'Rourke RA, Dell'Italia LJ (2004) Diagnosis and management of right ventricular myocardial infarction. Curr Probl Cardiol 29(1): 6-47.

8. Swan HJ, Ganz W, Forrester J, Marcus H, Diamond G, et al. (1970) Catheterization of the heart in man with use of a flow-directed balloontipped catheter. N Engl J Med 283(9): 447-451.

9. Dell'Italia LJ, Starling MR, O’Rourke RA (1983) Physical examination for exclusion of hemodynamically important right ventricular infarction. Ann Intern Med 99(5): 608-611.

10. Arrigo M, Huber LC, Winnik S (2019) Right Ventricular Failure: Pathophysiology, Diagnosis and Treatment. Card Fail Rev 5(3): 140-146.

11. Ostini F, Antoniazzi P, Pazin Filho A, Bestetti R, Cardoso MC, et al. (1998) 0 uso de drogas vasoativas em terapia intensiva. Medicina (Ribeirão Preto Online) 31(3): 400-411.

12. Cintron GB, Hernandez E, Linares E, Aranda JM (1981) Bedside recognition, incidence and clinical course of right ventricular infarction. Am J Cardiol 47(2): 224-227.

13. Konstam MA, Kiernan MS, Bernstein D, Bozkurt B, Jacob M, et al. (2018) Evaluation and Management of Right-Sided Heart Failure: A Scientific Statement From the American Heart Association. Circulation 137(20): e578-e622.

14. Dandel M, Hetzer R (2018) Evaluation of the right ventricle by echocardiography: particularities and major challenges. Expert Rev Cardiovasc Ther 16(4): 259-275.

15. Gorter TM, Lexis CP, Hummel YM, Lipsic E, Nijveldt R, et al. (2016) Right Ventricular Function After Acute Myocardial Infarction Treated With Primary Percutaneous Coronary Intervention (from the Glycometabolic Intervention as Adjunct to Primary Percutaneous Coronary Intervention in ST-Segment Elevation Myocardial Infarction III Trial). Am J Cardiol 118(3): 338-344

16. Rajesh GN, Raju D, Nandan D, Haridasan V, Vinayakumar D, et al. (2013) Echocardiographic assessment of right ventricular function in inferior wall myocardial infarction and angiographic correlation to proximal right coronary artery stenosis. Indian Heart J 65(5): 522-528.

17. Roshdy HS, El-Dosouky II, Soliman MH (2018) High-risk inferior myocardial infarction: Can speckle tracking predict proximal right coronary lesions. Clin Cardiol 41(1): 104-110.
18. Cabin HS, Clubb KS, Wackers FJ, Zaret BL (1987) Right ventricular myocardial infarction with anterior wall left ventricular infarction: an autopsy study. Am Heart J 113(1): 16-23.

19. Lopez-Sendon J, Coma-Canella I, Gamallo C (1981) Sensitivity and specificity of hemodynamic criteria in the diagnosis of acute right ventricular infarction. Circulation 64(3): 515-525.

20. Bowers TR, O’Neill WW, Grines C, Pica MC, Safian RD, et al. (1998) Effect of reperfusion on biventricular function and survival after right ventricular infarction. N Engl J Med 338(14): 933-940.

21. Goldstein JA, Vlahakes GJ, Verrier ED, Schiller NB, Botvinick E, et al (1983) Volume loading improves low cardiac output in experimental right ventricular infarction. J Am Coll Cardiol 2(2): 270-278.

22. Goldstein JA, Vlahakes GJ, Verrier ED, Schiller NB, Tyberg JV, et al (1982) The role of right ventricular systolic dysfunction and elevated intrapericardial pressure in the genesis of low output in experimental right ventricular infarction. Circulation 5(3): 513-522.

23. Shah AD, Patel AU, Knezevic A, Hoskins MH, Hirsh DS, et al. (2017) Clinical Performance of Magnetic Resonance Imaging Conditional and Nonconditional Cardiac Implantable Electronic Devices. Pacing Clin Electrophysiol 40(5): 467-475.

24. Dell'Italia LJ, Starling MR, Blumhardt R, Lasher JC, O'Rourke RA (1985) Comparative effects of volume loading, dobutamine, and nitroprusside in patients with predominant right ventricular infarction. Circulation 72(6): 1327-1335.

25. Siniorakis EE, Nikolaou NI, Sarantopoulos CD, Sotirelos KT, Iliopoulos $\mathrm{NE}$, et al. (1994) Volume loading in predominant right ventricular infarction: bedside haemodynamics using rapid response thermistors. Eur Heart J 15(10): 1340-1347.

26. Kapur NK, Paruchuri V, Korabathina R, Al-Mohammdi R, Mudd JO, et al (2011) Effects of a percutaneous mechanical circulatory support device for medically refractory right ventricular failure. J Heart Lung Transplant 30(12): 1360-1367.

27. Belohlavek J, Rohn V, Jansa P, Tosovsky J, Kunstyr J, et al. (2010) Venoarterial ECMO in severe acute right ventricular failure with pulmonary obstructive hemodynamic pattern. J Invasive Cardiol 22(8): 365-369.

28. Berman M, Tsui S, Vuylsteke A, Klein A, Jenkins DP (2008) Lifethreatening right ventricular failure in pulmonary hypertension: RVAD or ECMO. J Heart Lung Transplant 27(10): 1188-1189. 